\title{
Deregulation of miR-324/KISS1/kisspeptin in early ectopic pregnancy: mechanistic findings with clinical and diagnostic implications
}

\author{
Antonio Romero-Ruiz $\mathrm{PhD}^{\mathrm{a}, \mathrm{b}, \mathrm{c}}$, Maria $\mathrm{S}$. Avendaño $\mathrm{PhD}^{\mathrm{a}, \mathrm{b}, \mathrm{c}}$, Francisco \\ Dominguez $\mathrm{PhD}^{\mathrm{e}, \mathrm{f}}$, Teresa Lozoya $\mathrm{MD}, \mathrm{PhD}^{\mathrm{e}}$, Helena Molina-Abril $\mathrm{PhD}^{\mathrm{a}, \mathrm{g}}$, \\ Susana Sangiao-Alvarellos $\mathrm{PhD}^{\mathrm{b}, \mathrm{h}}$, Marta Gurrea $\mathrm{MD}, \mathrm{PhD}^{\mathrm{e}}$, Maribel Lara-Chica \\ $\mathrm{PhD}^{\mathrm{a}, \mathrm{b}, \mathrm{c}}$, Manuel Fernandez-Sanchez MD, $\mathrm{PhD}^{\mathrm{i}}$, Encarnación Torres-Jimenez \\ $\mathrm{BSc}^{\mathrm{a}, \mathrm{b}, \mathrm{c}}$, Cecilia Perdices-Lopez BSc ${ }^{\mathrm{a}, \mathrm{b}, \mathrm{c}}$, Ali Abbara MD, $\mathrm{PhD}^{\mathrm{j}}$, Liliana Steffani \\ $\mathrm{PhD}^{\mathrm{b}}$, Marco A. Calzado $\mathrm{PhD}^{\mathrm{a}, \mathrm{b}, \mathrm{c}}$, Waljit S. Dhillo MD, $\mathrm{PhD}^{\mathrm{j}}$, Antonio Pellicer \\ $\mathrm{MD}, \mathrm{PhD}^{\mathrm{e}}$, Manuel Tena-Sempere MD, $\mathrm{PhD}^{\mathrm{a}, \mathrm{b}, \mathrm{c}, \mathrm{d}, \mathrm{k}}$
}

\footnotetext{
${ }^{a}$ Instituto Maimónides de Investigación Biomédica de Cordoba (IMIBIC), Cordoba, Spain

${ }^{b}$ Department of Cell Biology, Physiology and Immunology, University of Cordoba, Cordoba, Spain

${ }^{c}$ Hospital Universitario Reina Sofia, Cordoba, Spain

${ }^{d}$ CIBER Fisiopatología de la Obesidad y Nutrición, Instituto de Salud Carlos III, Cordoba, Spain

${ }^{e}$ Instituto Valenciano de Infertilidad (IVI), University of Valencia, Valencia, Spain

${ }^{f}$ Instituto de Investigación Sanitaria Hospital Clínico de Valencia INCLIVA, Valencia, Spain

${ }^{g}$ Department of Applied Mathematics-I, University of Seville, Seville, Spain

${ }^{h}$ Instituto de Investigación Biomédica de A Coruña (INIBIC), A Coruña, Spain

${ }^{i}$ IVI-Sevilla, Sevilla, Spain

${ }^{j}$ Department of Investigative Medicine, Imperial College London, United Kingdom

${ }^{k}$ Institute of Biomedicine, University of Turku, Turku, Finland
} 


\begin{abstract}
BACKGROUND. Ectopic pregnancy is a life-threatening condition for which novel screening tools that would enable early accurate diagnosis would improve clinical outcomes. Kisspeptins, encoded by KISSI, play an essential role in human reproduction, at least partially by regulating placental function and possibly embryo implantation. Kisspeptin levels are elevated massively in normal pregnancy and reportedly altered in various gestational pathologic diseases. Yet, the pathophysiologic role of KISS1/kisspeptin in ectopic pregnancy has not been investigated previously.

OBJECTIVE. The purpose of this study was to evaluate changes of $K I S S 1 /$ kisspeptin levels in ectopic pregnancy and their underlaying molecular mechanisms and to ascertain the diagnostic implications of these changes.

STUDY DESIGN. A total of 122 women with normal pregnancy who underwent voluntary termination of pregnancy and 84 patients who experienced tubal ectopic pregnancy were recruited. Measurements of plasma kisspeptins and KISS1 expression analyses in human embryonic/placental tissue were conducted in ectopic pregnancy and voluntary termination of pregnancy control subjects during the early gestational window $(<12$ weeks). Putative microRNA regulators of KISS1 were predicted in silico, followed by expression analyses of selected microRNAs and validation of repressive interactions in vitro. Circulating levels of these microRNAs were also assayed in ectopic pregnancy vs voluntary termination of pregnancy.

RESULTS. Circulating kisspeptins gradually increased during the first trimester of normal pregnancy but were reduced markedly in ectopic pregnancy. This profile correlated with the expression levels of KISS1 in human embryonic/placental tissue, which increased in voluntary termination of pregnancy but remained suppressed in ectopic pregnancy. Bioinformatic predictions and expression analyses identified miR-27b-3p and miR-324-3p as putative repressors of KISS1 in human embryonic/placental tissue at $<12$ weeks gestation, when expression of microRNAs was low in voluntary termination of pregnancy control subjects but significantly increased in ectopic pregnancy. Yet, a significant repressive interaction was documented only for miR-324-3p, occurring at the predicted 3'-UTR of KISS1. Interestingly, circulating levels of miR-324-3p, but not of miR-27b-3p, were suppressed distinctly in ectopic pregnancy, despite elevated tissue expression of the pre-microRNA. A decision-tree model that used kisspeptin and miR-324-3p levels was successful in discriminating ectopic pregnancy vs voluntary termination of pregnancy, with a receiver-operating characteristic area under the curve of $0.95 \pm 0.02$ (95\% confidence interval).

CONCLUSION. Our results document a significant down-regulation of KISS1/kisspeptins in early stages of ectopic pregnancy via, at least partially, a repressive interaction with miR-324-3p. Our data identify circulating kisspeptins and miR-324-3p as putative biomarkers for accurate screening of ectopic pregnancy at early gestational ages.
\end{abstract}

Key words

Biomarker; diagnosis; ectopic pregnancy; KISS1; kisspeptins; miR-324-3p 
Ectopic pregnancy (EP) is defined as the implantation and development of a fertilized ovum elsewhere than in the uterine cavity. The fallopian tube is the most common site for ectopic implantation, ${ }^{1}$ which otherwise might occur also in the cervix, ovary, abdomen, or even a scar of a previous cesarean delivery. Approximately, $1.5-2 \%$ of all reported pregnancies are extrauterine. ${ }^{2}$ Despite substantial improvements in its timely management, EP remains the early pregnancy complication with the highest morbidity and mortality rates. ${ }^{2}$ EP can result in tubal rupture if not managed promptly, thereby compromising woman's health and future fertility. ${ }^{3}$ Although the overall incidence of EP increased by 6-fold between the 1970s and 1990s, its associated mortality rate has substantially fallen, largely because of better diagnosis and treatment before rupture. ${ }^{2}$ Nonetheless, EP still represents $9-13 \%$ of all pregnancy-related deaths in developed countries and is responsible for approximately $75 \%$ of deaths of the first trimester. The situation is much worse in developing countries; for instance, in Africa, women with EP encounter high fatality rates because of late diagnosis, where it accounts for up to $30 \%$ of pregnancy-associated deaths. ${ }^{4}$ Thus, there is an important clinical need to improve our understanding of the pathophysiologic basis of $\mathrm{EP}$ and to develop better tools for accurate early screening and diagnosis of this condition.

It is assumed that EP is multifactorial in origin, with several contributing factors, that include morphologic or functional alterations of fallopian tube permeability, perturbed chemotactic tubal environment, and/or deregulated tubal motility; the major risks factors for EP are a history of tubal surgery and/or extrauterine pregnancy. ${ }^{3}$ However, in more than one-half of cases of EP, no risk factor can be identified, which suggests the existence of additional, as yet unknown, underlying mechanisms. Current diagnosis of EP is based mainly on transvaginal ultrasonography, together with serial measurements of the $\beta$-subunit of human chorionic gonadotropin $(\beta-h C G)^{5}$, although laparoscopy is often needed as a method of certainty. Indeed, although ultrasound scans and $\beta$ hCG tests are extremely powerful for early detection of pregnancy and identification of some of its eventual complications, their sensitivity and specificity substantially decrease in the case of pregnancies of unknown location, ${ }^{3}$ in which false-positive or -negative diagnosis may occur. In this context, it is especially crucial to avoid erroneous diagnosis of a pregnancy as nonviable, because it may lead to medical or surgical interventions that could eliminate or severely damage a healthy pregnancy. ${ }^{5}$ Accordingly, different biomarkers have been proposed to increase the accuracy of EP diagnosis, which would allow a rapid triage of pregnancies at risk of extrauterine location. These putative markers may include circulating levels of progesterone, estradiol, vascular-endothelial growth factor-A, inhibin-A, and activin-A.6, 7, 8, 9, 10, 11 Yet, despite promising results, ${ }^{11}$ there is little pathogenic information emerging from these studies, and no diagnostic test based on these markers is currently available in clinical practice for accurate biochemical identification of EP.

Kisspeptins, the products of the KISS1 gene, have emerged in the last decade as master regulators of reproductive function in multiple species, including humans, ${ }^{12}$ as illustrated by the fact that patients with inactivating mutations of KISS1 or the cognate kisspeptin receptor, GPR54 $($ aka, KISS1R), experience severe hypogonadism of central origin.13, 14, 15 Despite the overwhelming evidence that supports a major function of brain (hypothalamic) kisspeptin signaling in the control of reproduction, mainly via its capacity to stimulate gonadotropinreleasing hormone neurons, ${ }^{16}$ additional functions of kisspeptins have been reported at peripheral levels of the reproductive axis, which includes the ovary and the uterus.17, 18 On the latter, mounting evidence has demonstrated that the elements of Kiss1 system are expressed in human endometrium and placenta, ${ }^{18}$ and kisspeptins have been proposed to play an important role in endometrial gland development and function ${ }^{19}$ and in human placentation.17, 18 Notably, expression and functional analyses have suggested that kisspeptin signaling is an important (negative) regulator of human trophoblast migration and invasion ${ }^{20}$ and a (positive) regulator of embryo implantation. ${ }^{18}$ Altogether, these findings attest a relevant function of kisspeptins in human placentation, which is further suggested by the fact that circulating levels of kisspeptins dramatically increase during gestation, ${ }^{21}$ with abundant expression of KISSI in human trophoblast, especially in the first trimester. ${ }^{20}$ Accordingly, inappropriately low kisspeptin levels have been proposed as a biomarker of gestational alterations, such as intrauterine growth restriction and 
preeclampsia,22, 23 and a putative predictor of miscarriage risk. ${ }^{24}$ In fact, a very recent study that compared a well-defined, as yet limited $(\mathrm{N}=20)$, population of women with viable intrauterine pregnancy vs women with confirmed spontaneous abortion suggested that kisspeptin levels during an early gestational window (weeks 6-10) might serve as a good biomarker of pregnancy viability. ${ }^{25}$ However, to our knowledge, no single study has addressed potential dynamic changes in $K I S S 1 /$ kisspeptin in EP and their eventual diagnostic utility in this condition.

The molecular mechanisms underlying EP remain largely unknown. Interestingly, recent studies have highlighted the potential contribution of deregulated microRNAs as putative pathogenic and/or diagnostic factors in EP. MicroRNAs are small, noncoding RNAs that can modulate (mainly repress) gene/protein expression, primarily via interaction with seed regions at the 3' untranslated region (3'-UTR) of target genes, thereby promoting messenger RNA (mRNA) degradation or preventing protein translation. ${ }^{26}$ Deregulation of microRNA expression in embryonic/placental tissues and the fallopian tube has been reported in EP.27, 28, 29 These studies have revealed changes in the expression patterns of microRNAs of the let7 family and miR-132, miR-145, miR149, miR-182, miR-196, miR-223, and miR-424 in ectopic implantation; yet, the putative mechanistic implications for these alterations are yet to be elucidated. In addition, changes in the circulating levels of some microRNAs have been associated to EP.30, 31 Thus, miR-323-3p reportedly was increased in women with EP, ${ }^{32}$ whereas additional studies have shown that circulating levels of miR-515-3p, miR-517a, miR-518b, miR-519d, miR-525-3p, and miR-873 are significantly lower in patients with ectopic implantation.32, 33, 34 Likewise, abnormal serum levels of miR-1247 and miR-1296a recently have been reported in patients with EP because of salpingitis. $^{35}$

Considering that microRNAs in blood are, in general, stable and relatively easy to detect, ${ }^{30}$ these data hold promise for the identification of a novel class of noninvasive biomarkers of EP. Yet, to date, no single diagnostic test based on the determination of microRNAs (alone or in combination) has been validated as reliable biomarker of EP. Moreover, despite recent evidence on the epigenetic control of hypothalamic Kiss 1 in rodents, ${ }^{36}$ to our knowledge, no single report has documented the existence of microRNA-mediated regulation of human KISSI.

In the aforementioned context, the aims of our work were (1) to evaluate potential changes in KISS1 mRNA expression (at embryonic/placental tissue) and circulating kisspeptin levels in EP and (2) to highlight eventual regulatory mechanisms of KISS1 expression in ectopic gestation, which involves microRNAs. We particularly have targeted an early gestational window $(<12$ weeks of gestation), which is a clinically relevant time-frame for the screening of patients for EP. 


\section{Methods}

\section{Ethical approval}

The present study was approved by the Institutional Review Board/Independent Ethics Committee of the Hospital Universitario La Fe, Valencia, Spain. Early embryonic/placental tissue (mostly trophoblast) was collected after obtaining the corresponding informed consent from each patient, as described elsewhere.28, 29

\section{Sample collection}

A total of 122 women with a normal ongoing pregnancy who desired a voluntary termination of pregnancy (VTOP) and 84 patients who experienced from tubal EP were recruited over a $>4$ year period, according to previous described criteria,28, 29 from whom plasma and/or embryonic/placental tissues were obtained. All individuals (EP and VTOP) included in this study signed an informed consent, and samples were dated properly according to the last menstrual period (week of pregnancy). Because of operational reasons, related with the sequential recruitment of cases over a protracted period of time by different personnel, it was not possible to obtain plasma and tissue samples, of sufficient quality for hormonal or molecular analyses, from all recruited patients. Likewise, because of limitations in the amount of the biologic materials that were sampled from some patients, not all analytes could be assayed in each sample, especially when different procedures (eg, radioimmunoassay vs quantitative polymerase chain reaction [qPCR]) were involved. Accordingly, the final group sizes per type of sample and analyte were (1) plasma kisspeptin (VTOP, 88; EP, 45), (2) plasma microRNAs (VTOP, 58; EP, 31), (3) tissue KISS1 (VTOP, 49; EP, 21), and (4) tissue microRNAs (VTOP, 90; EP, 35). Details about distribution of samples according to gestational age are provided in the Table.

The diagnosis of EP was based on clinical and physical examination, transvaginal ultrasound scans, and serial quantitative hCG levels and confirmed by laparoscopy, as described by Barnhart, ${ }^{1}$ in which the tissue was removed, in line with previously published procedures of our team.28, 29 The EP patients did not receive methotrexate treatment, and laparoscopy was performed in the following manner: ectopic pregnancies that were selected for this study were unruptured gestations located in the isthmus or the proximal ampulla. The tube that contained the EP was grasped at both sides (approximately $1 \mathrm{~cm}$ away from the gestation site), and bipolar coagulation was applied. Similarly, the adjacent mesentery was also coagulated. Then, salpingectomy was performed with the use of scissors, as standard procedure to avoid recurrence of EP; a longitudinal antimesenteric incision into the surface of the tube was made, and mild pressure was applied with 2 fingers to extract the gestational sac. Embryonic tissue was carefully separated from obvious blood clots or tubal tissue in the operating room under a stereomicroscope and was immediately placed in TRIzol reagent, frozen, and stored at $-80^{\circ} \mathrm{C}$ until use. A piece of each sample was sent to the Pathology Department (Hospital Universitario La Fe, Valencia) to provide histologic confirmation of EP and the absence of tubal tissue. The fetal dilation and evacuation method or fetal aspiration technique was performed in VTOP women to obtain the embryonic tissue. 


\section{RNA extraction and $q P C R$}

Total RNA was isolated from embryonic tissues and maternal plasma samples from different stages of pregnancy (both control and ectopic gestations), with the use of TRIzol reagent (Invitrogen, Carlsbad, CA), according to the manufacturer's protocol. The quality and concentration of the isolated RNA were determined by spectrophotometry, according to standard procedures. Real-time qPCR was performed on the samples with a Bio-Rad SFX 96 Real-Time System (Bio-Rad Laboratories, Hercules, CA), as described in detail elsewhere. ${ }^{37}$ For quantification of KISS1 mRNA in embryonic samples, $1 \mu \mathrm{g}$ of total RNA per tissue sample was treated with RQ1 RNAse-free DNAse-I (Promega, Madison, WY) and retro-transcribed in a $30 \mu \mathrm{L}$ reaction with the use of iScript Reverse Transcription Super-mix (Bio-Rad Laboratories). For realtime PCR amplification, we used SYBR Green qPCR Master Mix (Promega), with the following primer sequences: hKISS1-forward: 5'-AGC AGC TAG AAT CCC TGG G-3', position 1069810716 nt; hKISS1-reverse: 5'-AGG CCG AAG GAG TTC CAG T-3', position 10947-10929 nt. The primer pair: hL19-forward: 5'-GAA ATC GCC AAT GCC AAC TC-3' and hL19-reverse: 5'ACC TTC AGG TAC AGG CTG TG-3' was used for amplification of a 290-base pair fragment of the mRNA of ribosomal L19 protein, which served as internal control for reaction efficiency and sample loading. PCR was initiated by 1 cycle of $95^{\circ} \mathrm{C}$ for 2 minutes, followed by 35 cycles of 15 seconds at $95^{\circ} \mathrm{C}, 30$ seconds at $62^{\circ} \mathrm{C}$, and 10 seconds at $72^{\circ} \mathrm{C}$, followed by 1 final cycle of $72^{\circ} \mathrm{C}$ for 1 minute. Relative KISS $1 \mathrm{mRNA}$ levels were normalized against the expression levels of L19 transcript.

For microRNA analyses in embryonic tissue and plasma samples, quantification of the expression levels of miR-324-3p and miR-27b-3p was performed according to "miRCURY LNA Universal RT microRNA PCR individual assay - Instruction manual v6.2", as instructed by Exiqon (Vedbaek, Denmark). All microRNAs were reverse transcribed into complementary DNA in a single reaction step. The complementary DNA synthesis control (UniSp6) was added in the reverse transcription reaction, which gave us the opportunity to evaluate the retro-transcribed reaction. Complementary DNA and SYBR Green qPCR Master Mix (Promega) were transferred to the qPCR plate with specifics primers (Exiqon).

The final qPCR expression data for both mRNA (KISS1) and microRNA were obtained by the $2^{\Delta \Delta \mathrm{Ct}}$ method, according to the original qPCR equation $=2^{-[\Delta \mathrm{Ct} \text { reference } \Delta \mathrm{Ct} \text { target }]}$; the cycle threshold values from the $\leq 6$ week normal pregnancy group were used as reference, to provide relative units of expression. The same method was used to calculate the values of housekeeping internal control, L19 (mRNA) or U6 (microRNAs) in case of tissue samples and hsa-miR-191-5p for plasma. Finally, the ratio between sample and housekeeping was calculated to express relative units/internal control. For presentation, the final data were multiplied by 100 .

Note that, because of the differences in the length and nucleotide composition of the transcripts to be amplified and in the chemistry used for effective PCR amplification, technically reliable detection of microRNAs was achieved in a higher number of placental samples than KISSI (Table 1). 
Table. Group sizes and gestational age distribution for the different tissues and analytes assayed in the cohorts of women with normal and ectopic pregnancies

\begin{tabular}{|c|c|c|c|c|c|c|c|c|}
\hline \multirow{2}{*}{$\begin{array}{l}\text { Gestational } \\
\text { week }\end{array}$} & \multicolumn{2}{|c|}{ Plasma Kisspeptin } & \multicolumn{2}{|c|}{ Plasma microRNAs } & \multicolumn{2}{|c|}{ Tissue KISS1 } & \multicolumn{2}{|c|}{ Tissue microRNAs } \\
\hline & $\begin{array}{l}\text { Normal } \\
\text { pregnancy }\end{array}$ & $\begin{array}{l}\text { Ectopic } \\
\text { pregnancy }\end{array}$ & $\begin{array}{l}\text { Normal } \\
\text { pregnancy }\end{array}$ & $\begin{array}{l}\text { Ectopic } \\
\text { pregnancy }\end{array}$ & $\begin{array}{l}\text { Normal } \\
\text { pregnancy }\end{array}$ & $\begin{array}{l}\text { Ectopic } \\
\text { pregnancy }\end{array}$ & $\begin{array}{l}\text { Normal } \\
\text { pregnancy }\end{array}$ & $\begin{array}{l}\text { Ectopic } \\
\text { pregnancy }\end{array}$ \\
\hline$\leq 6$ & 10 & 27 & 9 & 16 & 6 & 13 & 9 & 16 \\
\hline 7 & 18 & 8 & 9 & 4 & 4 & 3 & 11 & 9 \\
\hline 8 & 12 & 5 & 10 & 5 & 8 & 2 & 13 & 3 \\
\hline $9-12$ & 25 & 6 & 13 & 6 & 17 & 3 & 25 & 7 \\
\hline Total $\leq 6-12$ & 65 & 45 & 41 & 31 & 35 & 21 & 58 & 35 \\
\hline $13-15$ & 14 & - & 9 & - & 10 & - & 20 & - \\
\hline $16-17$ & 6 & - & 5 & - & 2 & - & 7 & - \\
\hline $18-20$ & 3 & - & 3 & - & 2 & - & 5 & - \\
\hline TOTAL & 88 & 45 & 58 & 31 & 49 & 21 & 90 & 35 \\
\hline
\end{tabular}

Note: For operational reasons related with the sequential recruitment of cases over a protracted period of time by different personnel, it was not possible to obtain plasma and tissue samples of sufficient quality for hormonal or molecular analyses from all recruited patients. Likewise, because of limitations in the amount of the biologic materials that were sampled from some patients, not all analytes could be assayed in each sample, especially when involving different analytical procedures. The numbers in the Table correspond to effective individual analyses that were conducted per group and yielded technically reliable results.

Romero-Ruiz et al. Kisspeptin and miR-324-3p in ectopic pregnancy. Am J Obstet Gynecol 2019.

\section{Bioinformatic analysis}

Computational microRNA target prediction algorithms were applied to propose putative interactions between the UTR of KISS1 and validated human microRNAs in available databases. Algorithms for the prediction of microRNAs, which can putatively regulate KISSI, were applied to the 3'-UTR and 5' promoter regions, with the following inclusion criteria: (1) microRNAs predicted for interaction at the 3'-UTR had to be identified at least in 2 of the following databases: TargetScan (http://www. targetscan.org/; miRWalk (http://zmf.umm.uniheidelberg.de/apps/zmf/mirwalk2/); MicroCosm (http://www.ebi.ac. uk/enrightsrv/microcosm/htdocs/targets/v5/); or miRanda (http://www. microrna.org/microrna/ home/); (2) microRNAs predicted for interaction at the promoter region had to be identified with the use of the database miRWalk (http://mirwalk.umm.uni-heidelberg.de); and (3) for selected targets, conservation of at least 7 nucleotides at the seed region, complementary with KISS1 sequence, was required.

\section{Luciferase reporter assays}

HEK-293T cells (human embryonic kidney cells) were maintained in Dulbecco modified Eagle medium that was supplemented with $10 \%$ fetal bovine serum, $2 \mathrm{mmol} / \mathrm{L}$ L-glutamine, and $1 \%$ (volume/volume) penicillin/streptomycin at $37^{\circ} \mathrm{C}$ in a humidified atmosphere that contained $5 \%$ $\mathrm{CO}_{2}$. Transient transfections were performed with Rotifect (Carl Roth, Karlsruhe, Germany) according to manufacturer's instructions. The expression vectors for Gluc-KISS1-3'UTR reporter, hsa-miR-324-3p, hsa-miR-27b-3p, and related reagents were purchased from GeneCopoeia (Rockville, MD); luciferase assay was performed with Secret-Pair Dual Luminescence Assay Kit according to the manufacturer's instructions (Promega). For KISS1-3'UTR reporter assays, HEK293 T cells were co-transfected with KISS1-3'UTR reporter and hsa-miR-324-3p expression 
vectors. For KISS1 promoter analyses, cells were co-transfected with a KISS1-promoter-Gluc reporter plasmid (KISS1p1339; a gift from Dr Sabine Heger, University of Hanover, Germany) and the hsa-miR-27b-3p expression vector. A schematic of the 3'-UTR and 5'-UTR KISS1 reporter plasmids is presented in Supplemental Figure S1. As control subjects, cells co-transfected with the reporter vectors (KISS1-3'UTR or KISS1p1339) together with microRNA empty vectors or vectors harboring corresponding scramble hsa-miR sequences were included in all assays. Cells were collected in phosphate-buffered saline solution after 24 hours and lysed according to the instructions of the luciferase assay kit. Luciferase activity was measured with an Autolumat LB 9510 (EG\&G Berthold, Bad Wildbad, Germany).

\section{Assays for kisspeptins and total hCG}

Plasma kisspeptin levels were assayed in control (VTOP) and EP samples with a validated radioimmunoassay;38, 39 collection of blood samples was done in strict adherence to conditions known to preserve kisspeptins for proper immunometric detection. ${ }^{40}$ The kisspeptin antibody, GQ2, which was used at a final dilution of 1:3.500.000, has $100 \%$ cross-reactivity with human kisspeptin-54 and shorter fragments (kisspeptin-14 and -10), but $<0.01 \%$ with any other related human RFamide peptides. ${ }^{39}$ Kisspeptin-54 was labeled with ${ }^{125} \mathrm{I}$ by the Iodogen (Lund, Sweden) method. The assay was performed in duplicate with the use of dilutions of neat plasma in $0.7 \mathrm{~mL}$ of $0.06 \mathrm{M}$ phosphate buffer, with $0.3 \%$ bovine serum albumin, incubated for 3 days at $4{ }^{\circ} \mathrm{C}$. Free and antibody-bound labels were then separated by charcoal adsorption. The assay detected changes of $2 \mathrm{pmol} / \mathrm{L}$ of plasma kisspeptin immunoreactivity with a 95\% confidence limit. The intra- and interassay coefficients of variation were 8.3 and $10.2 \%$, respectively.

For comparative purposes, the levels of total hCG were also assayed in a subgroup of the VTOP and all available EP samples from the cohort in which plasma measurements of kisspeptins were conducted. The hCG assay was carried out according to the instructions of Human hCG ELISA Kit from Thermo-Fisher Scientific (Catalogue No\# EHCG). In this research (nonclinical) assay, the capture antibody detects the $\alpha$-subunit and cross-reacts with the whole molecule of hCG, and the detection antibody detects the $\beta$-subunit and cross-reacts with the whole molecule of hCG; this assay expresses results as picograms per milliliter units, which can be converted into milliinternational units per milliliter, considering an activity of the standard of the curve of 17.100 IU per mg, as reported by the manufacturer. This assay was applied to a subset of women with the VTOP group, during the first trimester of pregnancy, as control subjects ( $n=35$ women) and to the whole cohort of patients who experienced tubal EP ( $\mathrm{n}=45$ women).

\section{Statistical analyses}

Data of microRNA and KISS1/kisspeptin levels are expressed as medians with their interquartile range. A nonparametric Mann-Whitney test was used to evaluate differences between case and control groups (single comparisons). Analysis of variance was used for statistical analyses of multiple datapoints; when appropriate, the post-hoc Fisher least significant difference test was applied to identify individual differences after multiple comparisons. For data that followed normal distribution, results are expressed as means \pm standard deviation or standard error of the mean; statistical differences were assessed with the use of 2-tailed Student $\mathbf{t}$ tests (single comparisons) or analysis of variance for statistical analyses of multiple datapoints, followed when appropriate by post-hoc, Newman-Keuls tests. All statistics were carried out with the use of Prism 7.0c (GraphPad Software, Inc, San Diego, CA). A probability value of <.05 was considered to be statistically significant. 
Data of patients that were collected during the first 12 weeks of gestation were used to test the ability of kisspeptins, miR324-3p, and total hCG to predict EP. Different combinations of these variables were used after logarithmic transformation to build logistic regression models. Because of the limited number of cases, data were not divided into training and testing sets. On the contrary, models were built and validated with the use of repeated 10 -fold cross-validation. ${ }^{41} \mathrm{~K}$ fold cross-validation randomly divides the data into $k$ blocks of roughly equal size. Each block is left out in turn; the other $k-1$ blocks are used to train the model that is then used to predict the class of the remaining "left-out" subsample. Finally, classification results are summarized into performance measures and averaged to get the overall resampled estimate.

Area under the curve (AUC), sensitivity, specificity, accuracy (total correct predictions/total samples), and Cohen's (unweighed) Kappa statistic were computed for each variable's combination within the cross validation process (kisspeptin, miR324-3p, hCG, hCG/miR-324-3p, hCG/kisspeptin, and kisspeptin/miR-324-3p). Model performance was measured with the use of AUC, sensitivity, and specificity. Cutoff values were calculated with receiver-operating characteristic (ROC) analysis. Delong test was used to compare discriminatory ability across models. $^{42}$

According to the same validation procedure, a decision tree with the use of miR-324-3p and kisspeptin, with or without hCG, was built. The $\mathrm{C} 50$ method (an extension of the C4.5 classification algorithm described in https://github.com/topepo/caret/) was used for this purpose. All models were built and validated using the $\mathrm{R}$ package caret, as defined in http://www.rulequest.com/see5-unix.html.

\section{Results}

\section{Embryonic/placental tissue expression of KISS1 and circulating levels of kisspeptins in EP}

Dynamic profiles of embryonic/placental KISS1 expression and circulating levels of kisspeptins were compared between women with normal gestation who underwent VTOP (taken as control pregnancies) and women who experienced EP. For clinical reasons, we focused our analyses in a gestational window up to 12 weeks. For reference purposes, in the control group, additional samples from viable pregnancies up to 20 weeks of gestation were also included. Because of the limited number of samples available at early gestational ages and to increase statistical power, samples from 4,5 , and 6 weeks of gestation were grouped for analysis, as $\leq 6$ week sample. Likewise, in some analyses, data from VTOP samples at the intervals 7-9, 10-12, 13-15, and 16-17 weeks of gestation were pooled together. For the same reasons, samples from control pregnancies at or beyond week 18 of gestation were grouped as 18 - to 20 -week samples.

Analysis of KISSI gene expression in embryonic/placental tissue from women who underwent VTOP revealed negligible expression in $\leq 6$-week samples, with a significant increase thereafter, which reached a maximum $>10,000$-fold rise at week 12 of gestation. This was followed by a consistent, gradual decline in relative KISS1 levels from gestational week 13 onwards, with relative expression levels that had returned to $\leq 6$-week values in the $\geq 18$-week sample (Figure 1 , A). Circulating levels of kisspeptins followed a grossly similar profile during early gestation, with an ascending curve in the initial weeks of normal pregnancy and concentrations that increased gradually up to gestational week 15; yet, they remained at a plateau state thereafter (Figure 1, B). 


\section{TISSUE}

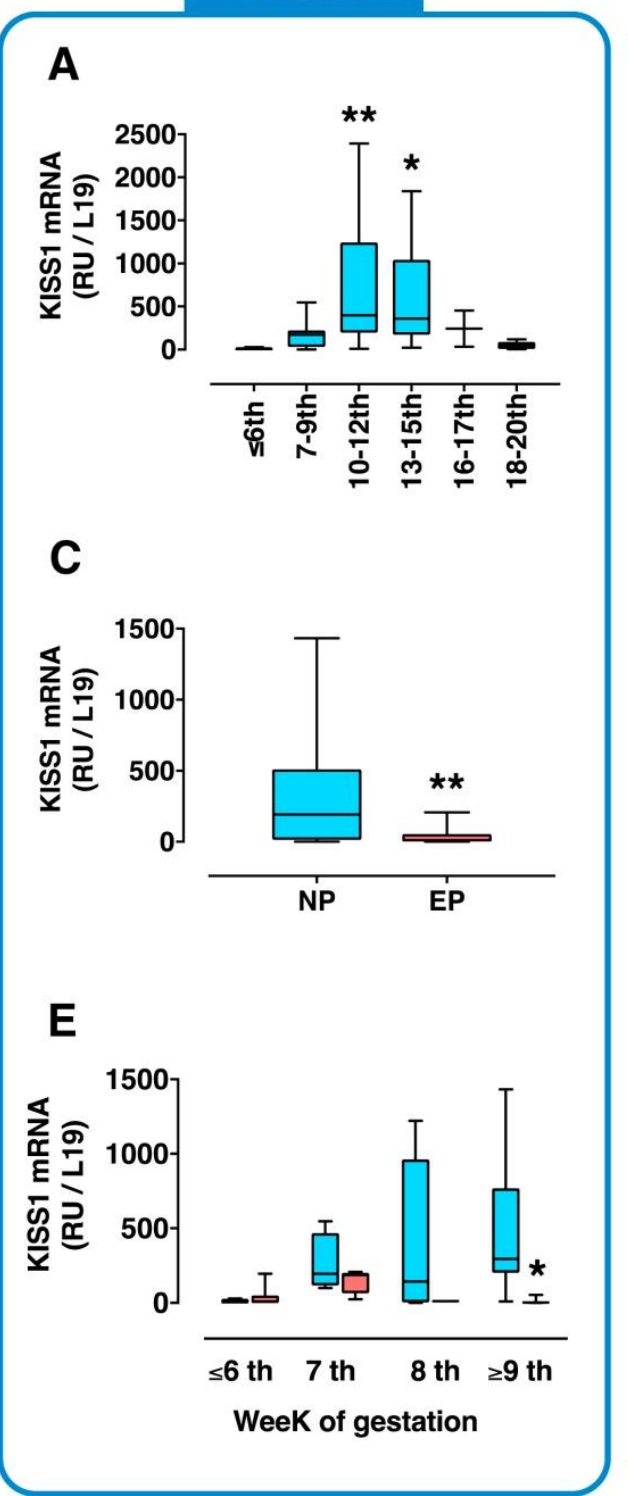

\section{PLASMA}

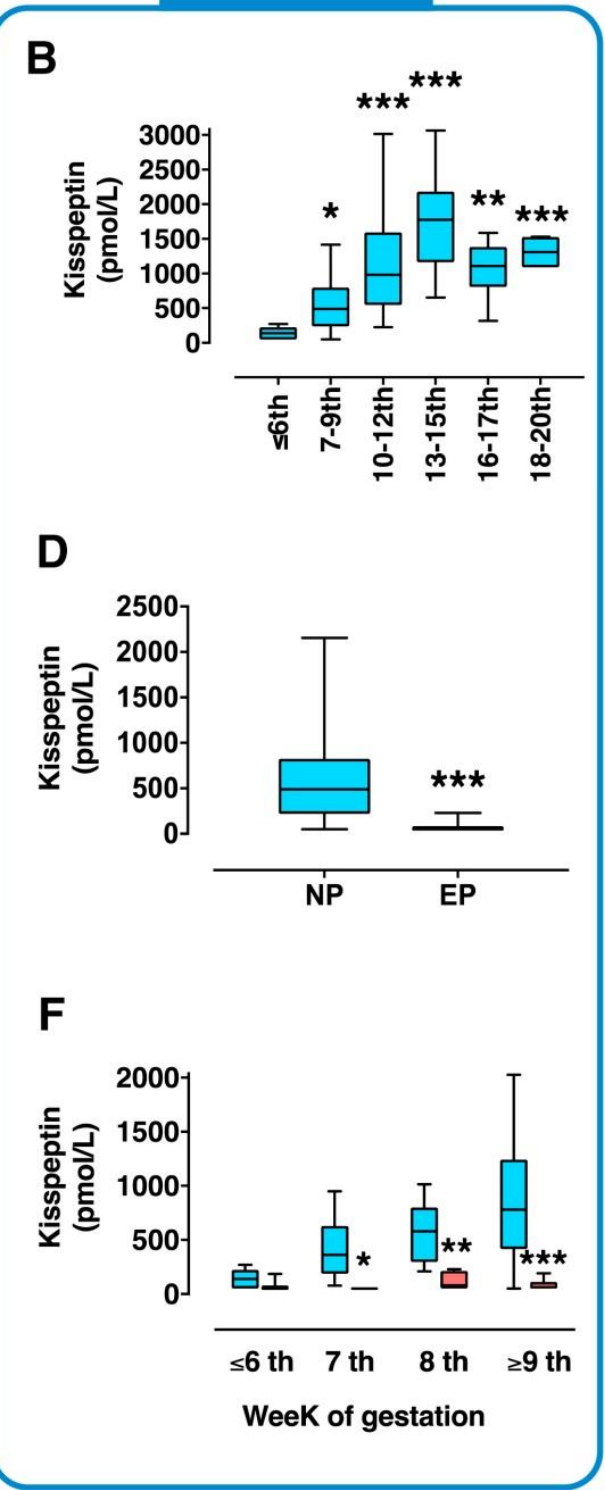

Figure 1. Expression of KISS1 in embryonic/placental tissue and plasma kisspeptin levels in normal and ectopic pregnancies

$\mathbf{A}$ and $\mathbf{B}$, Expression of KISS1 and kisspeptin levels in normal pregnancy (from voluntary termination of pregnancy) are grouped in 6 gestational age ranges: $\leq 6,7-9,10-12,13-15,16-17$, and 18-20 weeks of pregnancy. Analyses in ectopic pregnancies were restricted to samples collected until week 12 of ectopic gestation. C and D, Integral levels of KISS1 expression and plasma kisspeptin levels in samples from normal pregnancy and ectopic pregnancy up to week 12 of gestation are shown. $\mathbf{E}$ and $\mathbf{F}$, The detailed temporal course of these changes. Samples from weeks 5 and 6 of gestation were grouped as $\leq 6$ weeks, whereas those from weeks $9-12$ were grouped as $\geq 9$-week samples. Data are presented as medians and interquartile ranges. For presentation of KISS1 microRNA levels, data from the $\leq 6$-week normal pregnancy group were used as reference values to provide relative units of expression, which were normalized against the internal control, L19. One asterisk indicates $P<.05 ; 2$ asterisks indicate $P<.01$; and 3 asterisks indicate $P<.001$ vs corresponding values in normal pregnancy (Mann-Whitney test for single comparisons in panels $\mathbf{C}$ and $\mathbf{D}$ or analysis of variance followed by Fisher least significant difference test).

$E P$, ectopic pregnancy; $m R N A$, messenger RNA; $N P$, normal pregnancy; $R U$, relative units.

Romero-Ruiz et al. Kisspeptin and miR-324-3p in ectopic pregnancy. Am J Obstet Gynecol 2019. 
Similar analyses of KISSI expression and circulating kisspeptins were conducted in samples from women with EP, collected up to gestational week 12. When calculated as median and interquartile range values during the whole study period ( $<12$ week), both embryonic/placental KISS1 gene expression and circulating kisspeptin levels were significantly suppressed in women with EP vs VTOP (Figure 1, C-D). To precisely monitor the timing of such suppression, data analysis was split in $\leq 6,7,8$, and $\geq 9$ weeks of gestation. In terms of KISS1 mRNA in embryonic/placental tissue, expression levels were equally negligible in the $\leq 6$-week groups from VTOP and EP; yet, from week 7 onward, control pregnancies showed higher KISS1 mRNA levels than EPs, although differences were in the limit of statistical significance up to week 9 of pregnancy (Figure 1, E). Circulating levels of kisspeptins followed globally similar profiles, although in this case, plasma concentrations of kisspeptins were significantly lower in EP groups all through the study period, from week 7 until $\geq 9$ weeks of pregnancy (Figure 1, F).

\section{Embryonic/placental tissue expression of miR-324-3p and miR-27b-3p in EP}

In an attempt to discover putative regulators that are responsible for the suppression of KISS1 expression in EP, bioinformatics was applied to identify potential microRNA regulators by identification of their corresponding seed regions in the 3'-UTR (or eventually promoter) of the KISS1 gene. By a search of different databases and implementation of appropriate tools, 3 major putative candidates were identified: miR-137-3p and miR-324-3p (for which 8 and 7 nucleotides, respectively, from their predicted seed regions were found in the 3'-UTR of KISS1) and miR-27b$3 \mathrm{p}$, with 7 nucleotides of its seed region complementary to KISS1 promoter sequence (Supplemental Figure S1). Expression analyses subsequently were applied to evaluate the levels of these microRNAs at early stages of gestation. Our analyses documented that, although miR-324$3 p$ and miR-27b-3p are detectable readily in human embryonic/placental tissue during the first trimester of gestation, miR-137 is not, which therefore suggests negligible expression of this microRNA, which was excluded from further analyses.

Detailed expression analyses in embryonic/placental tissue from control pregnancies during the first 20 weeks of gestation demonstrated roughly similar expression profiles for both miR-324-3p and miR-27b-3p, with rather low relative expression levels during early stages of gestation, and a significant increase after week 13 (miR-324-3p) or week 16 (miR-27b-3p) of pregnancy (Figure 2, A-B). Similar analyses revealed that the expression levels of both microRNAs were elevated significantly in embryonic/placental tissue from EP that, when calculated as median values during the whole study period (<12 weeks of gestation), represented a 4-fold (miR-324-3p) and 3-fold (miR-27b-3p) increase vs VTOP levels (Figure 2, C-D). Timed analysis of such differences revealed that elevated expression of both microRNAs mainly concentrated at early stages, up to week 8 of gestation and were already significant at the <6-week group (Figure 2, E-F). 


\section{EMBRYO/PLACENTAL TISSUE}

A

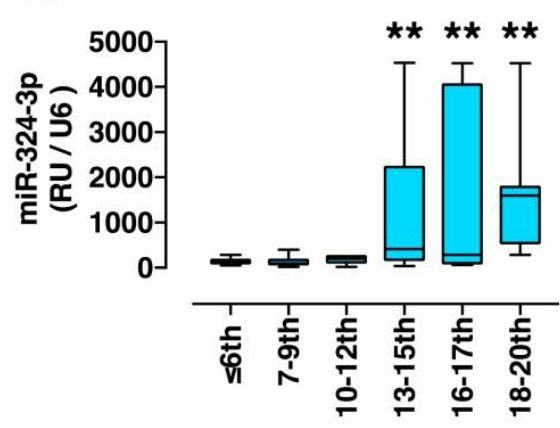

C

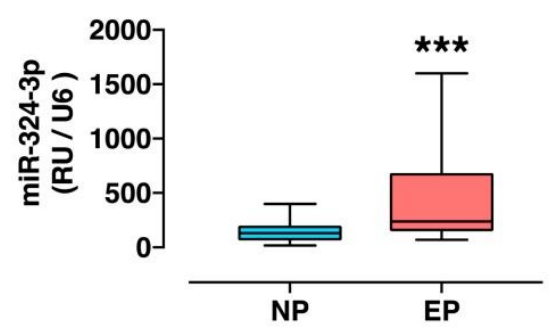

E

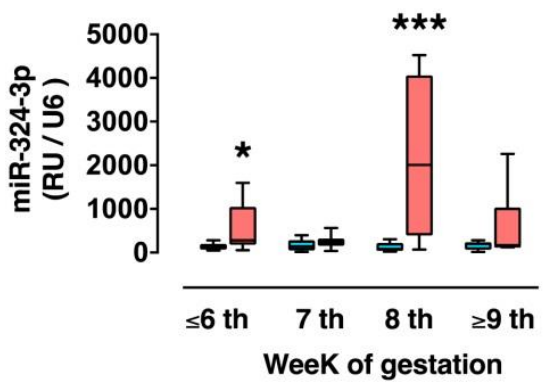

B

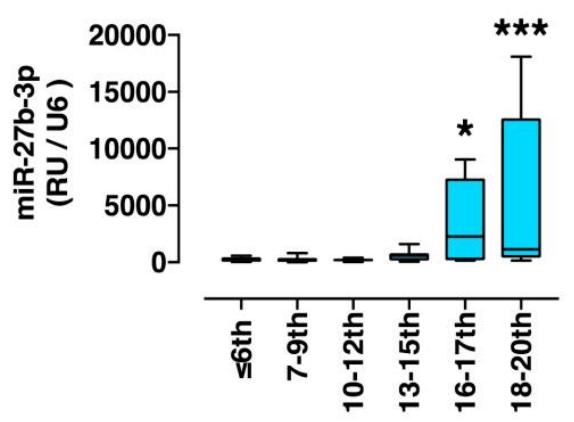

D

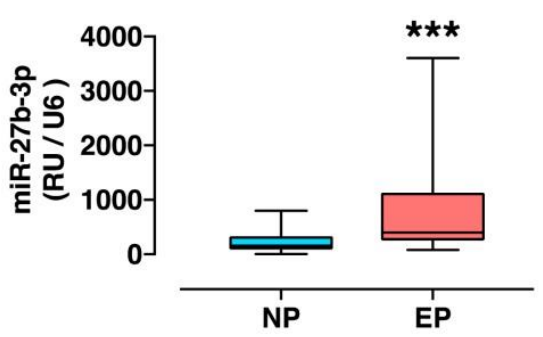

$\mathbf{F}$

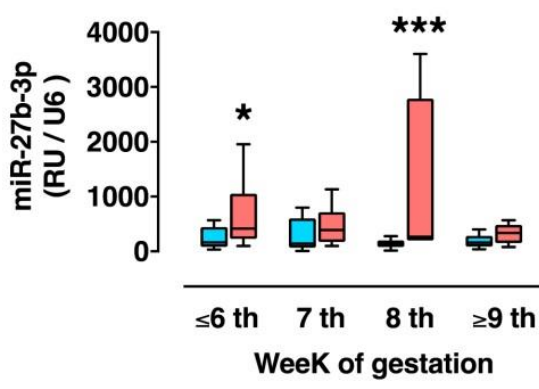

Figure 2. Expression of miR-324-3p and miR-27b-3p in embryonic/placental tissue in normal and ectopic pregnancies $\mathbf{A}$ and $\mathbf{B}$, Expression levels of miR-324-3p and miR-27b-3p in embryonic/placental tissue from control pregnancies (normal pregnancy; from voluntary termination of pregnancy) are presented, grouped in 6 gestational age ranges: $\leq 6,7-9,10-12$, $13-15,16-17$, and 18-20 weeks of pregnancy. Analyses in ectopic pregnancy were restricted to samples collected until week 12 of ectopic gestation. $\mathbf{C}$ and $\mathbf{D}$, Integral levels of miR-324-3p and miR-27b-3p in samples from normal pregnancy and ectopic pregnancy up to week 12 of gestation are shown. $\mathbf{E}$ and $\mathbf{F}$, The detailed temporal course of these changes is shown. Samples from week $5-6$ of gestation were grouped as $\leq 6$ week, whereas those from week $9-12$ were grouped as $>9$ week samples. Data are presented as medians and interquartile ranges. For presentation, data from the $\leq 6$-week normal pregnancy group were used as reference values to provide relative units of expression, which were normalized against the internal control, U6. One asterisk indicates $P<.05 ; 2$ asterisks indicate $P<.01$; and 3 asterisks indicate $P<.001$ vs corresponding values in normal pregnancy (Mann-Whitney test for single comparisons in panels $\mathbf{C}$ and $\mathbf{D}$ or analysis of variance followed by Fisher least significant difference test).

$E P$, ectopic pregnancy; $m R N A$, messenger RNA; $N P$, normal pregnancy; $R U$, relative units.

Romero-Ruiz et al. Kisspeptin and miR-324-3p in ectopic pregnancy. Am J Obstet Gynecol 2019. 
Based on bioinformatic predictions on the location of seed regions of miR324-3p and miR27b$3 \mathrm{p}$ at the 3'- and promoter region of the KISS1 gene, respectively, and the reciprocal changes in expression levels of these microRNAs and their putative target (KISS1/kisspeptins) in EP, we sought to demonstrate whether a direct repressive interaction actually exists, using proper luciferase reporter assays in vitro. For testing miR-324-3p/KISS1 interactions, HEK-293T cells were co-transfected with Gluc-KISSI-3'UTR and miR-324-3p reporter plasmids; the former harboring the KISS1 3'-UTR that contains the putative miR324-3p seed region downstream the coding sequence of luciferase. As shown in Figure 3, A, over-expression of miR324-3p induced a significant $30 \%$ drop in luciferase activity, therefore demonstrating a direct interaction and negative posttranscriptional regulation of KISS1 gene by miR-324-3p at its 3'-UTR. In contrast, given the predicted location of the seed region of miR-27b-3p at the promoter of KISSI, a reporter construct harboring the human KISS1 promoter region upstream, the luciferase coding sequence was used. HEK-293T cells were co-transfected with KISS1-promoter-GLuc and miR-27b-3p expression vectors. However, as shown in Figure 3, B, no significant changes in luciferase activity were found after miR-27b-3p over-expression in this heterologous cell system. An integrative model that depicts the putative repressive mechanism where-by miR-324-3p, but not miR-27b-3p, suppresses KISS1/kisspeptin expression is shown in Figure 3, C. 


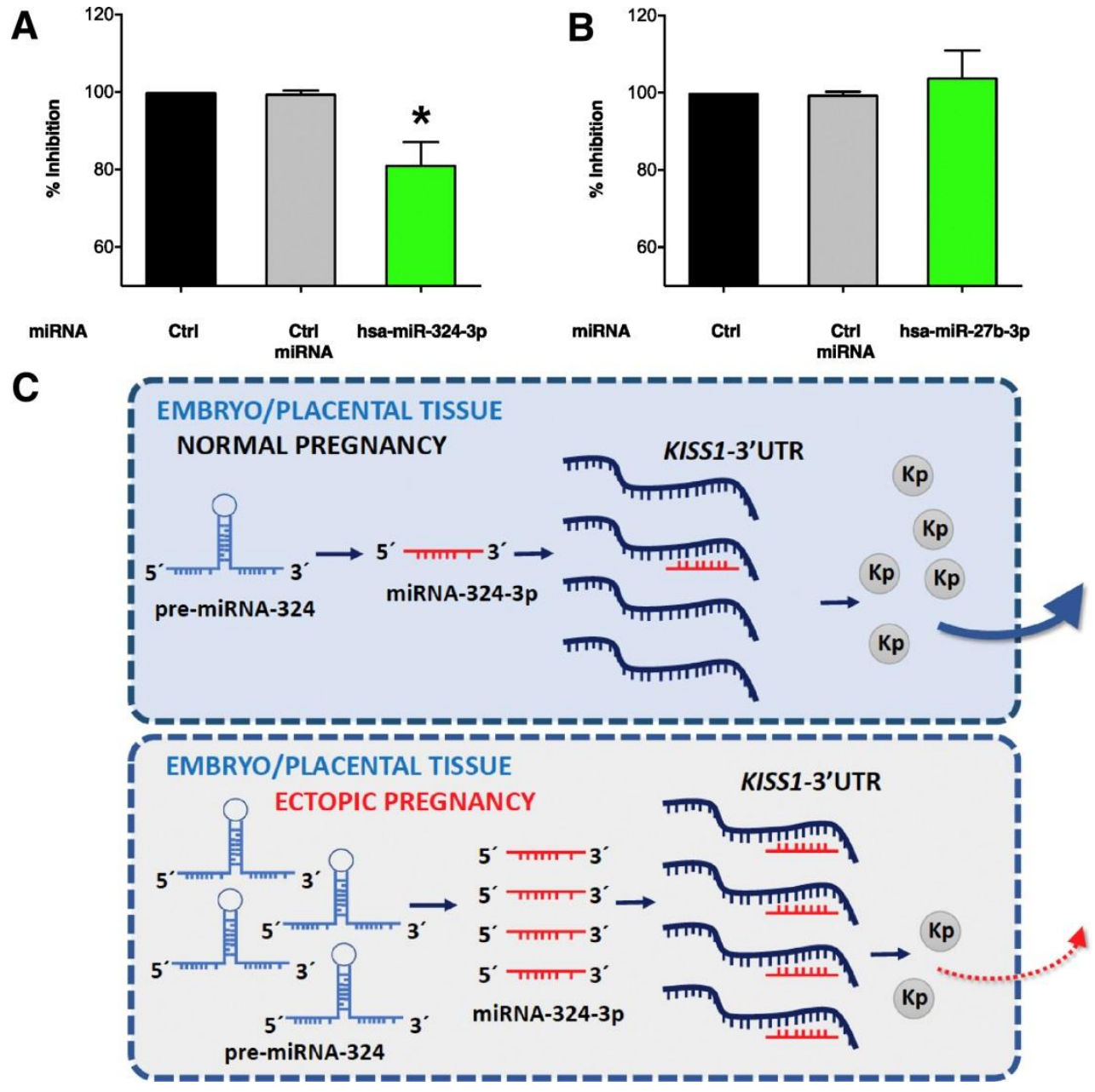

Figure 3. Potential interaction of miR-324-3p and miR-27b-3p with KISS1 in vitro

HEK-293T cells were co-transfected with 1 of the following reporter plasmid: A, KISS1-3'UTR-Gluc or B, KISS1promoter-GLuc together with a plasmid encoding the indicated microRNA (miR-324-3p) or miR-27b-3p or a microRNA scrambled control. Luciferase activity was measured as relative light units and is presented for each microRNA as percentage of inhibition with respect to control values (ie, cells transfected with the corresponding reporter plasmid together with the microRNA empty vector). As additional control, data from HEK-293T cells co-transfected with scramble has-miR vectors are also shown. Data are presented as the mean \pm standard deviation of $n=3$ experiments, which were done in triplicate. The asterisk indicates $P<.05$ (analysis of variance followed by Newman-Keuls test). C, A schematic is shown that depicts the proposed repressive mechanism, whereby miR-324-3p, acting at specific seed regions of the 3'-UTR of KISS1, suppresses expression, most likely by preventing its translation into kisspeptin protein. This would result in lowering of kisspeptin export to plasma (arrows) as seen in ectopic pregnancy conditions. Enhanced miR-324-3p in ectopic pregnancy is the consequence of increased expression of its pre-microRNA.

Ctrl, control; $K p$, kisspeptin; miRNA, microRNA.

Romero-Ruiz et al. Kisspeptin and miR-324-3p in ectopic pregnancy. Am J Obstet Gynecol 2019. 
Collectively, our results suggested that miR-324-3p and miR-27b-3p are deregulated in EP and that these changes (especially for miR-324-3p) may be relevant mechanistically for the observed suppression of KISS1/kisspeptin in ectopic gestations. To ascertain whether tissue alterations translate into detectable changes in the circulating levels of these microRNA, qPCR analyses were applied to plasma samples from VTOP and EP during the early gestational window. Circulating levels of miR-324-3p and miR-27b-3p were detectable in control (VTOP) pregnant women all through the study period, up to week 20 of gestation, with rather stable values (Figure 4, A-B); only a marginal increase in miR-324-3p levels was detected in 16- to 17-week samples (Figure 4, A). 


\section{PLASMA}

A
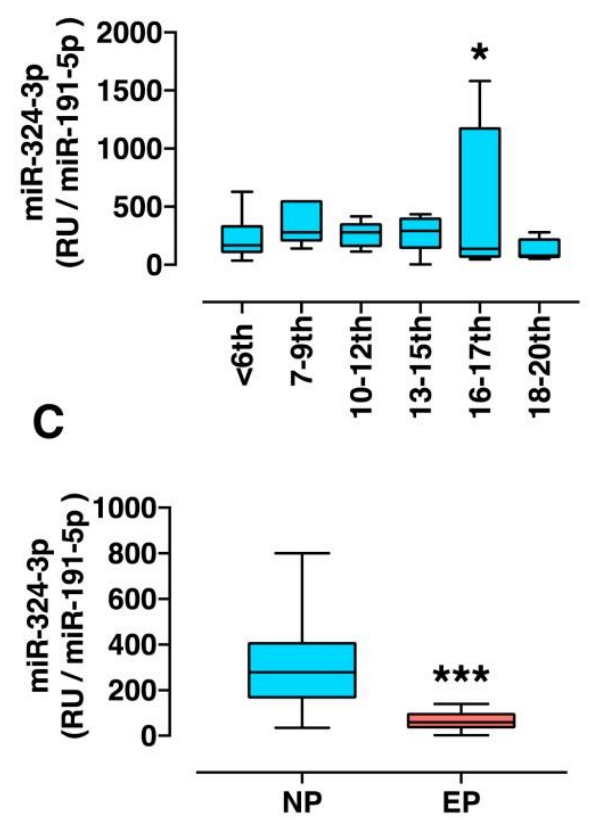

E

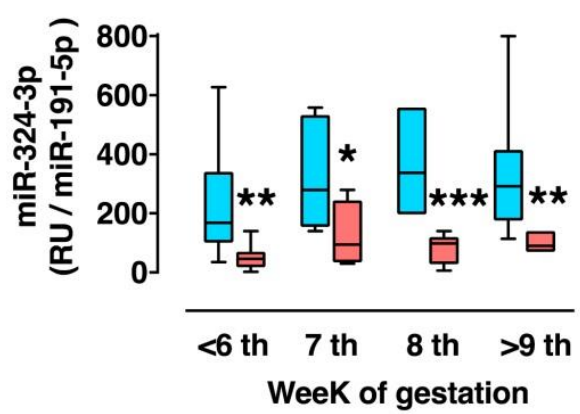

B
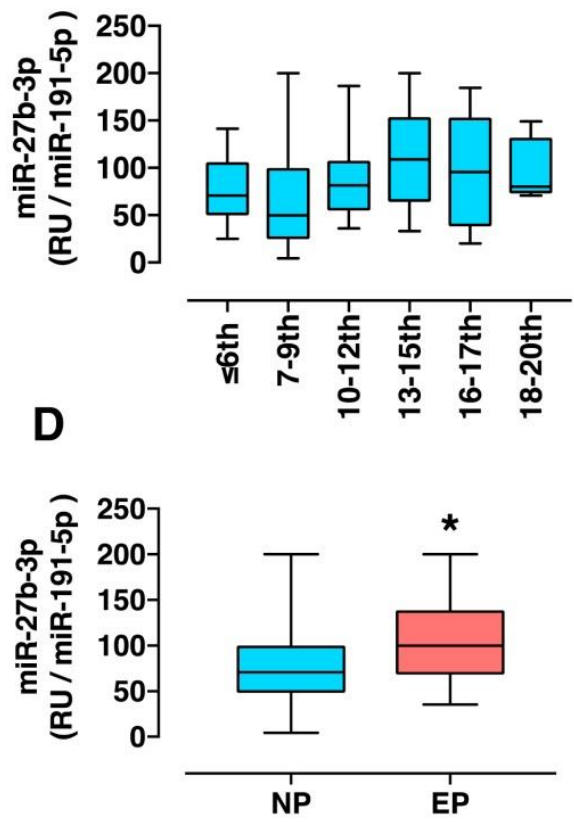

$\mathbf{F}$

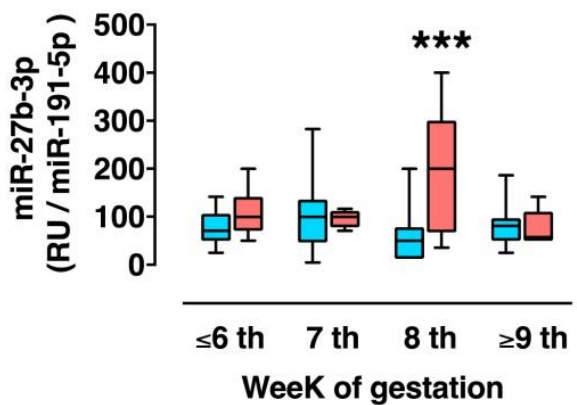

Figure 4. Plasma levels of miR-324-3p and miR-27b-3p in normal and ectopic pregnancies

$\mathbf{A}$ and B, Plasma levels of miR-324-3p and miR-27b-3p in samples from control pregnancies (normal pregnancy; from voluntary termination of pregnancy) are presented, grouped in 6 gestational age ranges: $\leq 6,7-9,10-12,13-15,16-17$, and 18-20 weeks of pregnancy. Analyses in ectopic pregnancy were restricted to samples collected until week 12 of ectopic gestation. C and D, Integral plasma levels of miR-324-3p and miR-27b-3p in samples from normal and ectopic pregnancies up to week 12 of gestation are shown. $\mathbf{E}$ and $\mathbf{F}$, The detailed temporal course of these changes is shown. Samples from week 5-6 of gestation were grouped as $\leq 6$ week, whereas those from weeks $9-12$ were grouped as $\geq 9$-week samples. Data are presented as medians and interquartile ranges. For presentation, data from the $\leq 6$-week normal pregnancy group were used as reference values to provide relative units of expression, which were normalized against the internal control, miR191-5p. One asterisk indicates $P<.05 ; 2$ asterisks indicate $P<.01$; and 3 asterisks indicate $P<.001$ vs corresponding values in normal pregnancy (Mann-Whitney test for single comparisons in panels $\mathbf{C}$ and $\mathbf{D}$ or analysis of variance followed by Fisher least significant difference test).

$E P$, ectopic pregnancy; $N P$, normal pregnancy; $R U$, relative units.

Romero-Ruiz et al. Kisspeptin and miR-324-3p in ectopic pregnancy. Am J Obstet Gynecol 2019. 
Integral plasma levels of miR-324-3p, calculated as median and interquartile range values during the <12-week period, were suppressed significantly in EP vs corresponding VTOP levels, with a marked $80 \%$ drop in mean concentrations (Figure 4, C). This drop is in contrast with the observed increase in tissue levels of miR324-3p in EP, which clearly are elevated. To further document this discrepancy, the expression levels of the precursor, pre-miR324-3p, were assayed in $<12$-week samples from VTOP and EP. In line with the expression profile of mature microRNA, pre-miR324-3p levels in EP were increased significantly (Supplemental Figure S2), therefore confirming the divergence between tissue expression and circulating levels of this microRNA. Opposite to miR-324-3p, mean plasma levels of miR-27b-3p were increased moderately in EP during the $<12$-week period (Figure 4, D), which parallels the expression data of the mature microRNA.

Timed analysis of the aforementioned changes revealed that circulating levels of miR-324-3p are suppressed consistently in EP all through the early gestational window (grouped at $\leq 6-$, 7-, 8-, and $\geq 9$-week samples), with statistically significant difference being detected from week 6 onwards (Figure 4, E). In contrast, no significant changes in the plasma levels of miR-27b-3p were detected between control and EP, except for a transient increase in EP at gestational week 8 (Figure 4, F).

\section{Circulating kisspeptin/miR-324-3p as new early biomarker of EP}

Based on the aforementioned data, biostatistics tools were used to study the usefulness of circulating kisspeptins and miR-324-3p as early diagnostic biomarkers of EP, measured alone or in combination. For validation purposes, hCG levels, as gold-standard for biochemical diagnosis of EP, were also measured in a representative set of VTOP and EP patients of our cohort. As shown in Supplemental Figure S3, total hCG concentrations were suppressed markedly in EP vs VTOP, when calculated as median levels over the $<12$-week gestational period, which is a profile that resembles that of kisspeptins and miR-324-3p. Time analysis at the $\leq 6,7,8$, and $\geq 9$ weeks of gestation revealed similar differences between control and EP, with a consistent suppression of hCG concentrations in EP patients, at all time-points studied.

Logistic regressions were applied to evaluate the prognostic power of kisspeptin, miR-324-3p, total hCG, and their combinations in the prediction of EP, during the first 12 weeks of gestation. Logistic regression models were built with the use of the $\mathrm{R}$ package caret and validated with repeated 10-fold cross-validation. Prediction scores demonstrated the capacity of kisspeptins and miR-324-3p to discriminate correctly between EP and VTOP groups. Cross-validation and observed model performances are shown in Figure 5, A. Interestingly, the predictive power of the combination of kisspeptins/miR-324-3p was similar to that of hCG (Delong test; $P=.6567$ ), and aggregation of miR-324-3p and hCG yielded ROC values close to the unit. 
A ROC Curve
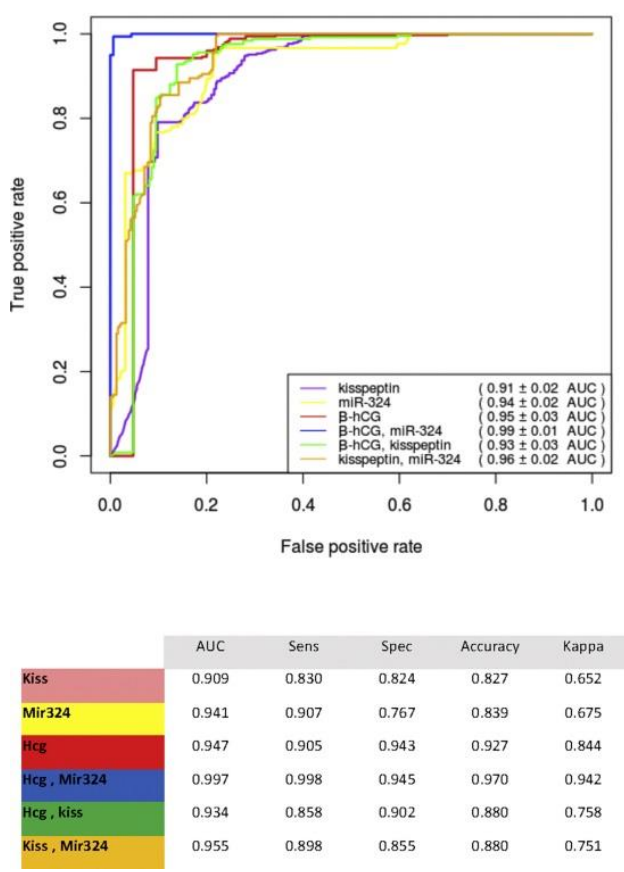

B

\section{Tree-based Decision Model}

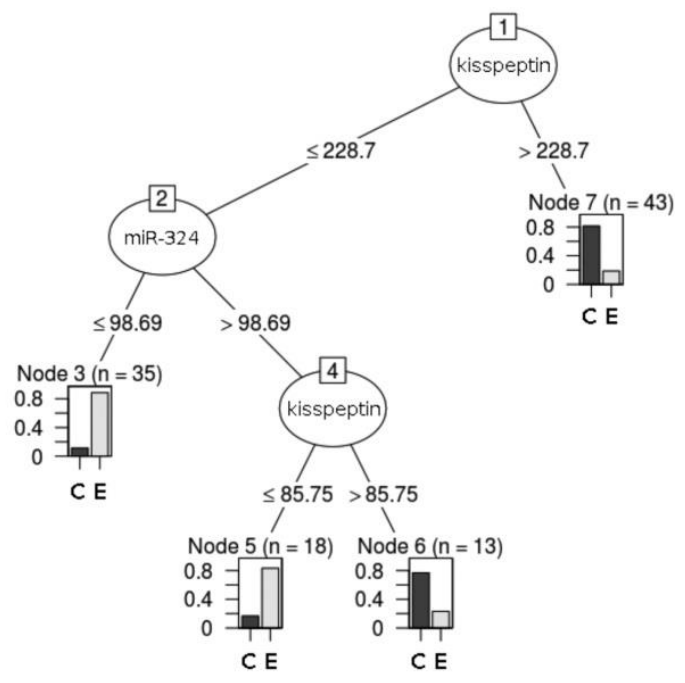

Figure 5. Plasma kisspeptin and miR-324-3p levels as diagnostic markers of ectopic pregnancy

A, Receiver-operating characteristic curve analyses of kisspeptins, miR-324-3p, total human chorionic gonadotropin, and their combinations in predicting ectopic pregnancy. Lower left panel, Performance measures of the corresponding logistic regression models are presented. B, A decision-tree model that was generated with the use of the miR324-3p and kisspeptin levels for the prediction of ectopic pregnancy is shown.

$C$, control pregnancy; $E$, ectopic pregnancy; $h C G$, human chorionic gonadotropin; $R O C$, receiver-operating characteristic. Romero-Ruiz et al. Kisspeptin and miR-324-3p in ectopic pregnancy. Am J Obstet Gynecol 2019.

Based on these findings, a decision-tree model that used miR-324-3p and kisspeptin levels was constructed with the use of the $\mathrm{J} 48$ method implemented in the R package caret and validated with repeated 10-fold cross-validation (AUC, $0.91 \pm 0.01 ; 95 \%$ confidence interval). In this model (Figure 5, B), the first node is based on kisspeptin levels $(\leq 228.7 \mathrm{pg} / \mathrm{mL}[\mathrm{n}=66]$ or $\geq 228.7$ $\mathrm{pg} / \mathrm{mL}[\mathrm{n}=43])$; the second node is based on miR-324-3p levels $(\leq 98.69$ counts, $n=35$, or $\geq 98.59$ counts, $\mathrm{n}=31)$, and the third node is based on kisspeptin levels $(\leq 85.75 \mathrm{pg} / \mathrm{mL}[\mathrm{n}=18]$ or $\leq 85.75$ $\mathrm{pg} / \mathrm{mL}[\mathrm{n}=13]$ ). In addition, with a similar approach, a decision-tree model that also incorporated total hCG levels was built (Supplemental Figure S4). In this model, the first node is based on hCG levels (threshold: $15,424 \mathrm{mIU} / \mathrm{mL}$ ), with a second level of discrimination for values below hCG threshold based on kisspeptin levels (threshold: $196.91 \mathrm{pg} / \mathrm{mL}$ ), and a third level based on miR324-3p (threshold: 43.46 counts). According to this model, for hCG levels over the threshold, discrimination is based on kisspeptin levels, with a nodal point at $85.75 \mathrm{pg} / \mathrm{mL}$. 


\section{Comment}

Although current strategies for detection of EP that involve transvaginal ultrasonography and serial determinations of $\beta$-hCG have proven reliable, false positives and negatives still occur. For instance, in nearly $20 \%$ of cases, EP patients display hCG profiles similar to those of intrauterine pregnancy; in $10 \%$ of cases, hCG levels resemble those of early miscarriage. ${ }^{3}$ This has prompted the search for novel markers of EP, which may improve the sensitivity and specificity of current protocols for early diagnosis. Yet, despite some promising findings, ${ }^{11}$ no alternative methods for biochemical triage of women at risk of EP at early stages of gestation are routinely in use in clinical practice. Likewise, the search for such novel biomarkers has not expanded substantially our understanding of the pathogenic mechanisms underlying ectopic placentation, which might help to define additional risk factors.

Human placentation shares some similarities with cancer migration and metastasis. ${ }^{43}$ In this context, kisspeptins, which initially were identified as potential metastasis-suppressing factors abundantly expressed in the placenta, were suspected to be important players for the fine control of trophoblast invasion and placentation.17, 18 This placental dimension of kisspeptins was further attested by the proven elevation of kisspeptin levels already at early stages of human gestation. ${ }^{21}$ Accordingly, a number of gestational conditions putatively that are linked to abnormal placentation that ranges from intrauterine growth restriction to preeclampsia have been associated with alterations in circulating kisspeptin levels.22, 23 Our current data unambiguously document, for the first time, that circulating kisspeptin levels are decreased markedly during the first trimester of gestation in EP, with a significant drop being already detectable at very early stages ( $\leq 6$ weeks of gestation). This profile grossly correlated with the expression levels of KISS1 in embryonic/placental tissue, in keeping with a potential placental source. Importantly, very few studies to date have correlated the expression profiles of KISS1 and plasma kisspeptin concentrations during the early gestational period; disparities between tissue expression and circulating levels have been described at later gestational ages in some diseases, such as preeclampsia. ${ }^{44}$ In our study, the observed drop in KISS1 expression might have an impact on the whole process of trophoblast invasion, which is inhibited by locally produced kisspeptins, 20, 45 and might facilitate nidation at an ectopic site. Kisspeptins have been suggested also to facilitate initial embryo adhesion/implantation at proper endometrial sites;18, 46 high tubal expression of Kiss1/kisspeptin has been proposed as a mechanism to prevent ectopic implantation in rats. ${ }^{47}$ Whether early deregulation of kisspeptin production might facilitate adherence at extrauterine sites in human pregnancy is yet to be elucidated.

All studies reported to date that have demonstrated alterations in kisspeptin levels or KISS1 expression were associative in nature and therefore did not provide mechanistic information for the observed changes. In contrast, our analyses also intended to identify potential mechanisms for deregulated KISS1/kisspeptin expression in EP. We particularly focused on the role of putative microRNA regulators of KISS1 that may contribute to these changes. Our rationale was double: (1) deregulated expression of some microRNAs has been linked to EP,27, 28, 29, 32, 33 and (2) microRNAs have a potential diagnostic dimension, because they are amenable for detection in blood samples. ${ }^{30}$ To our knowledge, our study is the first to address the potential direct regulation of KISS1 by microRNAs in a physiologic setting. Notably, despite recent evidence for epigenetic modulation of the KISS1 system in the brain, mainly in the context of pubertal maturation, ${ }^{48}$ the contribution of microRNA pathways in this phenomenon has not been documented to date. Based on a combination of bioinformatic and expression analyses, we identified 2 potential microRNA regulators of placental KISS1 expression, namely miR-27b-3p and miR-324-3p, because they were predicted to have conserved seed regions at the UTRs of the KISS1 gene and displayed detectable expression in embryonic/placental tissue during early gestational periods. Furthermore, opposite to KISS1 and kisspeptin levels, relative expression of both microRNAs was increased globally in EP during this period, which is compatible with the predicted repressive action of these microRNAs on the expression/translation of its target gene, KISS1. Admittedly, the tissue samples available did not permit discrimination of the specific cellular source of these microRNAs, which might operate 
intra- or even transcellularly. For similar reasons, we could not ascertain whether the elements of such microRNA/KISS1 system might be also expressed in the Fallopian tube, thus playing a role in the control of ectopic implantation, as previously suggested in a preclinical (rat) model. ${ }^{47}$

This putative regulatory role was further interrogated in vitro, with the use of appropriate reporter assays. Of note, although most microRNA-target repressive interactions take place at the $3^{\prime}$-UTR, ${ }^{26}$ microRNA-binding sites have been identified also at the promoter region of certain genes, which may drive suppressive or stimulatory effects on gene transcription.49, 50 This appeared to be the case for KISS1, for which a conserved seed region for miR-27b-3p was found in its promoter. However, luciferase promoter assays in HEK-293 cells failed to detect any significant impact of miR-27b-3p on KISS1 promoter activity, at least in our heterologous system. In clear contrast, over-expression of miR-324-3p in HEK-293 cells was capable of repressing KISS1 expression, as denoted by decreased luciferase activity that used an expression vector that included the 3'-UTR of KISS1. Given the heterologous nature of the assay, it remains possible that the net magnitude of the observed changes (roughly 30\% drop) might not be indicative of the real extent of this repressive interaction in vivo. In addition, our present results do not exclude the possibility of additional contributing factors for suppressed KISS1 expression in EP. Yet, our data unambiguously demonstrate, for the first time, the capacity of miR-324-3p to suppress KISS1, thus reinforcing the plausibility that the observed increase in miR-324-3p expression in ectopic embryonic/placental tissue is responsible, at least partially, for reduced expression of KISS1/kisspeptin levels in EP (Figure 3, C). Moreover, our findings collectively suggest that reduced kisspeptin levels in EPs are not merely due to defective placentation, but rather the consequence of an orchestrated deregulation of a miR-324-3p/KISS1 pathway in ectopic gestational tissue.

To explore the potential diagnostic value of altered expression of miR-324-3p and miR-27b-3p in EP, we also measured the circulating levels of both microRNAs. Surprisingly, although only a rather modest increase in circulating miR-27b-3p was detected in EP, which was significant only at week 8 of pregnancy, the circulating levels of miR-324-3p were clearly suppressed in ectopic gestations, with a significant reduction being already detectable at very early (<6-week) stages. The fact that not only the levels of this mature microRNA but also of its pre-microRNA were increased significantly in ectopic tissue suggests that the drop in circulating miR-324-3p concentrations in EP might be due to defective export of this microRNA from its embryonic/placental source, which may lead to tissue accumulation of miR-324-3p, which is a phenomenon that may further contribute, via repressive interaction with KISS1, to the reduction in circulating kisspeptins in EP. In any event, from a diagnostic standpoint, the concomitant marked suppression of kisspeptin and miR-324-3p levels offers diagnostic possibilities that were explored in our study. Thus, a biostatistical algorithm based on plasma levels of both factors allowed us to construct a decision-tree model that was successful in discriminating EP vs control pregnancy, with ROC AUC values that were roughly analogous to those offered by determination of hCG alone. Moreover, combination of total hCG with these novel factors, especially miR-324-3p, permitted us to further increase the discriminating power, with ROC data close to 1 , although incorporation of these complementary markers, namely kisspeptins and miR-324-3p, should nullify the risk of false positives or negatives. In fact, although suppressed kisspeptin levels have been associated with overall risk of miscarriage, ${ }^{24}$ to our knowledge, no study that has linked changes in circulating miR-324-3p with gestational alterations has been reported to date. All these features reinforce the potential of this method for biochemical triage of pregnancies that are at risk of ectopic gestation. Additional validation in independent cohorts and confirmation of the precise thresholds for our regression tree models are currently in progress to further refine the diagnostic protocol proposed herein. 
In sum, our data are the first conclusively to demonstrate a suppression of KISS1/kisspeptin levels in EP and to support the existence of a novel miR-324-3p/KISS1 regulatory pathway, which is altered and may have pathophysiologic implications during early EP. Our data also are endowed with a promising diagnostic dimension because, pending further validation, determination of kisspeptins and/or miR-324-3p, alone or in combination with hCG, might allow the improvement of current methods for noninvasive identification of EP, with superior sensitivity and specificity.

\section{References}

1. Barnhart KT. Clinical practice: ectopic pregnancy. N Engl J Med 2009;361:379-87.

2. Farquhar CM. Ectopic pregnancy. Lancet 2005;366:583-91.

3. Taran FA, Kagan KO, Hubner M, Hoopmann M, Wallwiener D, Brucker S. The diagnosis and treatment of ectopic pregnancy. Dtsch Arztebl Int 2015;112:693-703; quiz 4-5.

4. Goyaux N, Leke R, Keita N, Thonneau P. Ectopic pregnancy in African developing countries. Acta Obstet Gynecol Scand 2003;82:305-12.

5. Doubilet PM, Benson CB, Bourne T, et al. Diagnostic criteria for nonviable pregnancy early in the first trimester. N Engl J Med 2013;369:1443-51.

6. Majeed H, Hojgaard A, Johannesen P, Ladefoged ML, Forman A, Bor P. Predictive value of serum human chorionic gonadotropin ratio, progesterone and inhibin A for expectant management of early pregnancies of unknown location. Eur J Obstet Gynecol Reprod Biol 2012;165:66-9.

7. Guha S, Ayim F, Ludlow J, et al. Triaging pregnancies of unknown location: the performance of protocols based on single serum progesterone or repeated serum hCG levels. Hum Reprod 2014;29:938-45.

8. Zou S, Li X, Feng Y, et al. Comparison of the diagnostic values of circulating steroid hormones, VEGF-A, PIGF, and ADAM12 in women with ectopic pregnancy. J Transl Med 2013;11:44.

9. Feng C, Chen ZY, Zhang J, Xu H, Zhang XM, Huang XF. Clinical utility of serum reproductive hormones for the early diagnosis of ectopic pregnancy in the first trimester. J Obstet Gynaecol Res 2013;39:528-35.

10. Van Calster B, Bobdiwala S, Guha S, et al. Managing pregnancy of unknown location based on initial serum progesterone and serial serum hCG levels: development and validation of a twostep triage protocol. Ultrasound Obstet Gynecol 2016;48:642-9.

11. Rausch ME, Sammel MD, Takacs P, Chung K, Shaunik A, Barnhart KT. Development of a multiple marker test for ectopic pregnancy. Obstet Gynecol 2011;117:573-82.

12. Pinilla L, Aguilar E, Dieguez C, Millar RP, Tena-Sempere M. Kisspeptins and reproduction: physiological roles and regulatory mechanisms. Physiol Rev 2012;92:1235-316.

13. De Roux N, Genin E, Carel JC, Matsuda F, Chaussain JL, Milgrom E. Hypogonadotropic hypogonadism due to loss of function of the KiSS1-derived peptide receptor GPR54. Proc Natl Acad Sci U S A 2003;100:10972-6.

14. Seminara SB, Messager S, Chatzidaki EE, et al. The GPR54 gene as a regulator of puberty. N Engl J Med 2003;349:1614-27.

15. Topaloglu AK, Tello JA, Kotan LD, et al. Inactivating KISS1 mutation and hypogonadotropic hypogonadism. N Engl J Med 2012;366:629-35.

16. Avendano MS, Vazquez MJ, Tena-Sempere M. Disentangling puberty: novel neuroendocrine pathways and mechanisms for the control of mammalian puberty. Hum Reprod Update 2017;23:737-63.

17. Bhattacharya M, Babwah AV. Kisspeptin: beyond the brain. Endocrinology 2015;156:1218-27.

18. Babwah AV. Uterine and placental KISS1 regulate pregnancy: what we know and the challenges that lie ahead. Reproduction 2015;150:R121-8.

19. Leon S, Fernadois D, Sull A, et al. Beyond the brain-peripheral kisspeptin signaling is essential for promoting endometrial gland development and function. Sci Rep 2016;6:29073.

20. Bilban M, Ghaffari-Tabrizi N, Hintermann E, et al. Kisspeptin-10, a KiSS-1/metastin-derived decapeptide, is a physiological invasion inhibitor of primary human trophoblasts. J Cell Sci 2004;117:1319-28.

21. Horikoshi Y, Matsumoto H, Takatsu Y, et al. Dramatic elevation of plasma metastin concentrations in human pregnancy: metastin as a novel placenta-derived hormone in humans. $\mathrm{J}$ Clin Endocrinol Metab 2003;88:914-9.

22. Logie JJ, Denison FC, Riley SC, et al. Evaluation of kisspeptin levels in obese pregnancy as a biomarker for pre-eclampsia. Clin Endocrinol (Oxf) 2012;76:887-93. 
23. Armstrong RA, Reynolds RM, Leask R, Shearing CH, Calder AA, Riley SC. Decreased serum levels of kisspeptin in early pregnancy are associated with intra-uterine growth restriction and pre-eclampsia. Prenat Diagn 2009;29:982-5.

24. Jayasena CN, Abbara A, Izzi-Engbeaya C, et al. Reduced levels of plasma kisspeptin during the antenatal booking visit are associated with increased risk of miscarriage. J Clin Endocrinol Metab 2014;99:E2652-60.

25. Sullivan-Pyke C, Haisenleder DJ, Senapati S, et al. Kisspeptin as a new serum biomarker to discriminate miscarriage from viable intrauterine pregnancy. Fertil Steril 2018;109:137-41.e2.

26. Sayed D, Abdellatif M. MicroRNAs in development and disease. Physiol Rev 2011;91:827-87.

27. Feng Y, Zou S, Weijdegard B, et al. The onset of human ectopic pregnancy demonstrates a differential expression of miRNAs and their cognate targets in the Fallopian tube. Int J Clin Exp Pathol 2014;7:64-79.

28. Lozoya T, Dominguez F, Romero-Ruiz A, et al. The Lin28/Let-7 system in early human embryonic tissue and ectopic pregnancy. PLoS One 2014;9:e87698.

29. Dominguez F, Moreno-Moya JM, Lozoya T, et al. Embryonic miRNA profiles of normal and ectopic pregnancies. PLoS One 2014;9:e102185.

30. Cretoiu D, Xu J, Xiao J, Suciu N, Cretoiu SM. Circulating microRNAs as potential molecular biomarkers in pathophysiological evolution of pregnancy. Dis Markers 2016;2016:3851054.

31. Kontomanolis EN, Kalagasidou S, Fasoulakis Z. MicroRNAs as potential serum biomarkers for early detection of ectopic pregnancy. Cureus 2018;10:e2344.

32. Zhao Z, Zhao Q, Warrick J, et al. Circulating microRNA miR-323-3p as a biomarker of ectopic pregnancy. Clin Chem 2012;58:896-905.

33. Lu Q, Yan Q, Xu F, et al. MicroRNA-873 is a potential serum biomarker for the detection of ectopic pregnancy. Cell Physiol Biochem 2017;41:2513-22.

34. Miura K, Higashijima A, Mishima H, et al. Pregnancy-associated microRNAs in plasma as potential molecular markers of ectopic pregnancy. Fertil Steril 2015;103:1202-8.e1.

35. Zhang S, Sun Q, Jiang X, Gao F. Clinical significance of expression of hsa-mir-1247 and hsamir-1269a in ectopic pregnancy due to salpingitis. Exp Ther Med 2018;15:4901-5.

36. Lomniczi A, Wright H, Ojeda SR. Epigenetic regulation of female puberty. Front Neuroendocrinol 2015;36:90-107.

37. Sangiao-Alvarellos S, Manfredi-Lozano M, Ruiz-Pino F, et al. Changes in hypothalamic expression of the Lin28/let-7 system and related microRNAs during postnatal maturation and after experimental manipulations of puberty. Endocrinology 2013;154:942-55.

38. Dhillo WS, Chaudhri OB, Patterson M, et al. Kisspeptin-54 stimulates the hypothalamicpituitary gonadal axis in human males. J Clin Endocrinol Metab 2005;90:6609_ 15.

39. Dhillo WS, Savage P, Murphy KG, et al. Plasma kisspeptin is raised in patients with gestational trophoblastic neoplasia and falls during treatment. Am J Physiol Endocrinol Metab 2006;291:E878-84.

40. Ramachandran R, Patterson M, Murphy KG, et al. Preanalytical factors affecting RIA measurement of plasma kisspeptin. Clin Chem 2008;54:615-7.

41. Kim J-H. Estimating classification error rate: repeated cross-validation, repeated hold-out and bootstrap. Computational Statistics \& Data Analysis 2009;53:3735-45.

42. DeLong ER, DeLong DM, Clarke-Pearson DL. Comparing the areas under two or more correlated receiver operating characteristic curves: a nonparametric approach. Biometrics 1988;44:837-45.

43. Lorincz MC, Schubeler D. Evidence for converging DNA methylation pathways in placenta and cancer. Dev Cell 2017;43:257-8.

44. Matjila M, Millar R, van der Spuy Z, Katz A. Elevated placental expression at the maternalfetal interface but diminished maternal circulatory kisspeptin in preeclamptic pregnancies. Pregnancy Hypertens 2016;6:79-87.

45. Taylor J, Pampillo M, Bhattacharya M, Babwah AV. Kisspeptin/KISS1R signaling potentiates extravillous trophoblast adhesion to type-I collagen in a PKC- and ERK1/2-dependent manner. Mol Reprod Dev 2014;81:42-54.

46. Calder M, Chan YM, Raj R, et al. Implantation failure in female Kiss1-/- mice is independent of their hypogonadic state and can be partially rescued by leukemia inhibitory factor. Endocrinology 2014;155:3065-78.

47. Gaytan M, Castellano JM, Roa J, Sanchez-Criado JE, Tena-Sempere M, Gaytan F. Expression of KiSS-1 in rat oviduct: possible involvement in prevention of ectopic implantation? Cell Tissue Res 2007;329:571-9.

48. Lomniczi A, Ojeda SR. The emerging role of epigenetics in the regulation of female puberty. Endocr Dev 2016;29:1-16. 
49. Lytle JR, Yario TA, Steitz JA. Target mRNAs are repressed as efficiently by microRNAbinding sites in the 5' UTR as in the 3' UTR. Proc Natl Acad Sci U S A 2007;104:9667-72.

50. Orom UA, Nielsen FC, Lund AH. MicroRNA-10a binds the 5'UTR of ribosomal protein mRNAs and enhances their translation. Mol Cell 2008;30:460-71.

\section{Apppendix}

A

\begin{tabular}{|c|c|c|c|c|c|}
\hline Specie & mRNA & Region & miRNA & Seed Leght & Sequences \\
\hline Human & Kiss1 (5'-3') & $3^{\prime}$ UTR & miR-137-3p (3'-5') & 7 & $\begin{array}{c}5^{\prime} \text { GGAGCTTCCAACCCGAGGCAATAA 3' } \\
3^{\prime} \text { GAUGCGCAUAAGAAUUCGUAUU 5' }\end{array}$ \\
\hline Human & Kiss1 (5'-3') & $3^{\prime}$ UTR & miR-324-3p (3'-5') & 8 & $\begin{array}{l}5^{\prime} \text { GGGCGCAG-GTGCGGGGCAGTGAA 3' } \\
3^{\prime} \text { GGUCGUCGUGGACCCGUCACCC 5' }\end{array}$ \\
\hline Human & Kiss1 (5'-3') & Promoter & miR-27b (3'-5') & 8 & $\begin{array}{l}5^{\prime} \text { 'TAGCCCCTCTGCC--TTCA-GAGA 3' } \\
5^{\prime} \text { UUC-AC-AG-UGGCUAAGUUCUGC 3' }\end{array}$ \\
\hline
\end{tabular}

в

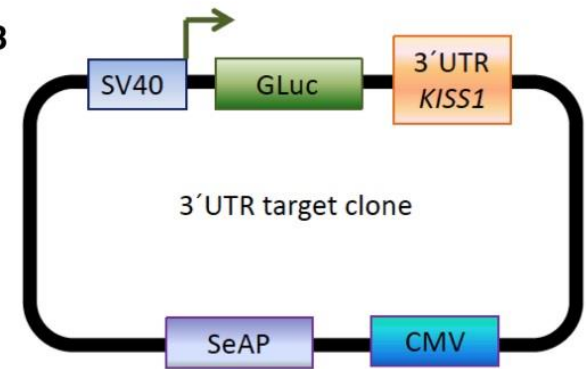

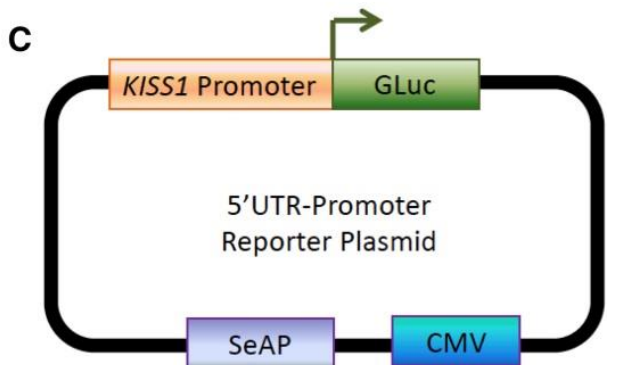

Supplemental Figure S1. Bioinformatic prediction of putative microRNA regulators of human KISS1 and reporter constructs for in vitro analyses

A, Bioinformatic tools were applied to identify conserved seed regions of microRNAs that may operate as regulators of KISS1. Three major candidates were identified: miR-324-3p and miR-137-3p (with predicted seed sequences at the 3'-UTR of $K I S S 1$ ) and miR-27b-3p (with a predicted seed region at the promoter of the gene). Details about location and sequence of these recognition sites are included. In addition, in the lower panels, schematics are shown of the reporter constructs that were used in vitro to explore the putative repressive interactions of miR-324-3p at the 3'-UTR (B, the KISS1-3'UTR-Gluc reporter vector) and of miR-27b-3p at the promoter (C, the KISS1-promoter-GLuc reporter vector) of KISS1.

$C M V$, cytomegalovirus; Gluc, gaussia luciferase; $S e A P$, secreted embryonic alkaline phosphatase; $S V 40$, simian virus 40 ; $U T R$, untranslated region.

Romero-Ruiz et al. Kisspeptin and miR-324-3p in ectopic pregnancy. Am J Obstet Gynecol 2019. 


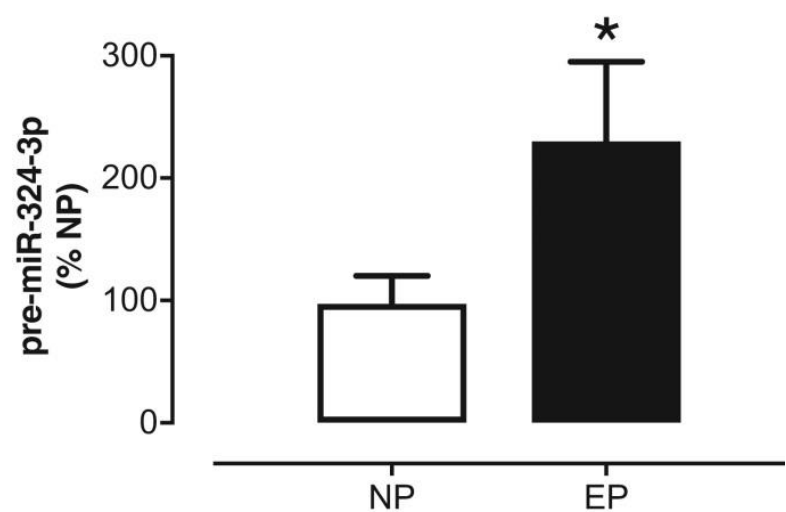

Supplemental Figure S2. Expression of pre-miR-324-3p in embryonic/placental tissue in normal and ectopic pregnancies Integral mean expression levels of pre-miR-324-3p in samples from normal pregnancy and ectopic pregnancy up to week 12 of gestation are shown; ectopic pregnancy values are expressed as normalized values against normal pregnancy levels. The asterisk indicates $P<.05$ (Student $t$ test).

$E P$, ectopic pregnancy; $N P$, normal pregnancy.

Romero-Ruiz et al. Kisspeptin and miR-324-3p in ectopic pregnancy. Am J Obstet Gynecol 2019. 


\section{PLASMA}

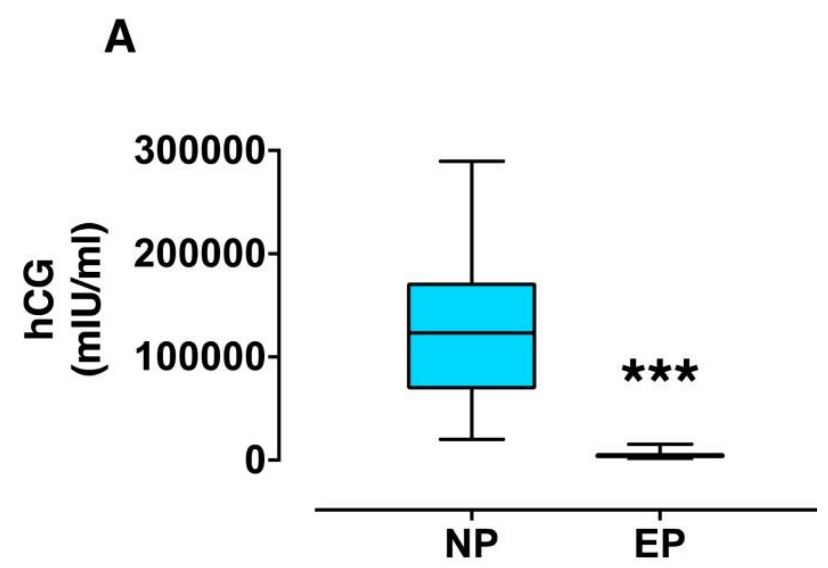

B

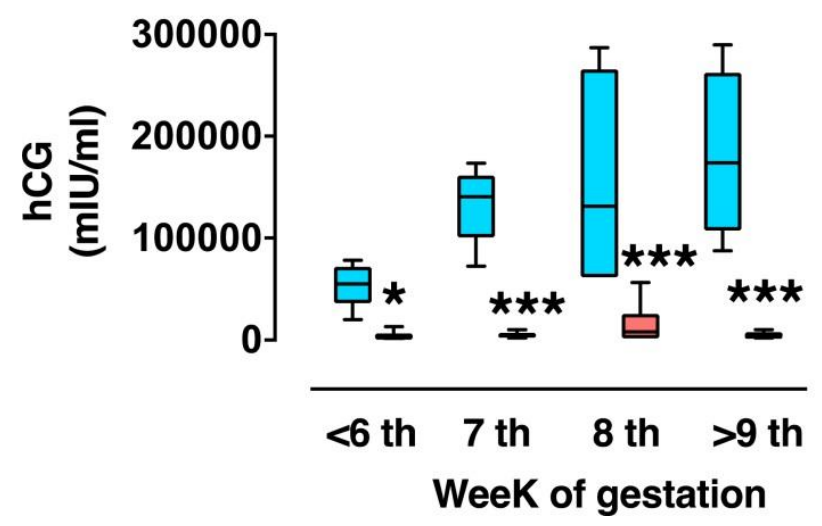

Supplemental Figure S3. Plasma levels of total human chorionic gonadotropin in normal and ectopic pregnancies

A, Integral plasma levels of human chorionic gonadotropin in samples from normal and ectopic pregnancies up to week 12 of gestation are shown; ectopic pregnancy values are expressed as normalized values against normal pregnancy levels. B The detailed temporal course of these changes is shown. Samples from week 5-6 of gestation were grouped as $\leq 6$ weeks samples, whereas those from week $9-12$ were grouped as $\geq 9$ week samples. Absolute levels are presented in milliinternational units per milliliters as medians and interquartile ranges. One asterisk indicates $P<.05$ and 3 asterisks indicate $P<.001$ vs corresponding values in normal pregnancy (A, Mann-Whitney test for single comparisons or $\mathbf{B}$, analysis of variance followed by Fisher least significant difference test).

$E P$, ectopic pregnancy; $h C G$, human chorionic gonadotropin; NP, normal pregnancy; $U T R$, untranslated region.

Romero-Ruiz et al. Kisspeptin and miR-324-3p in ectopic pregnancy. Am J Obstet Gynecol 2019. 


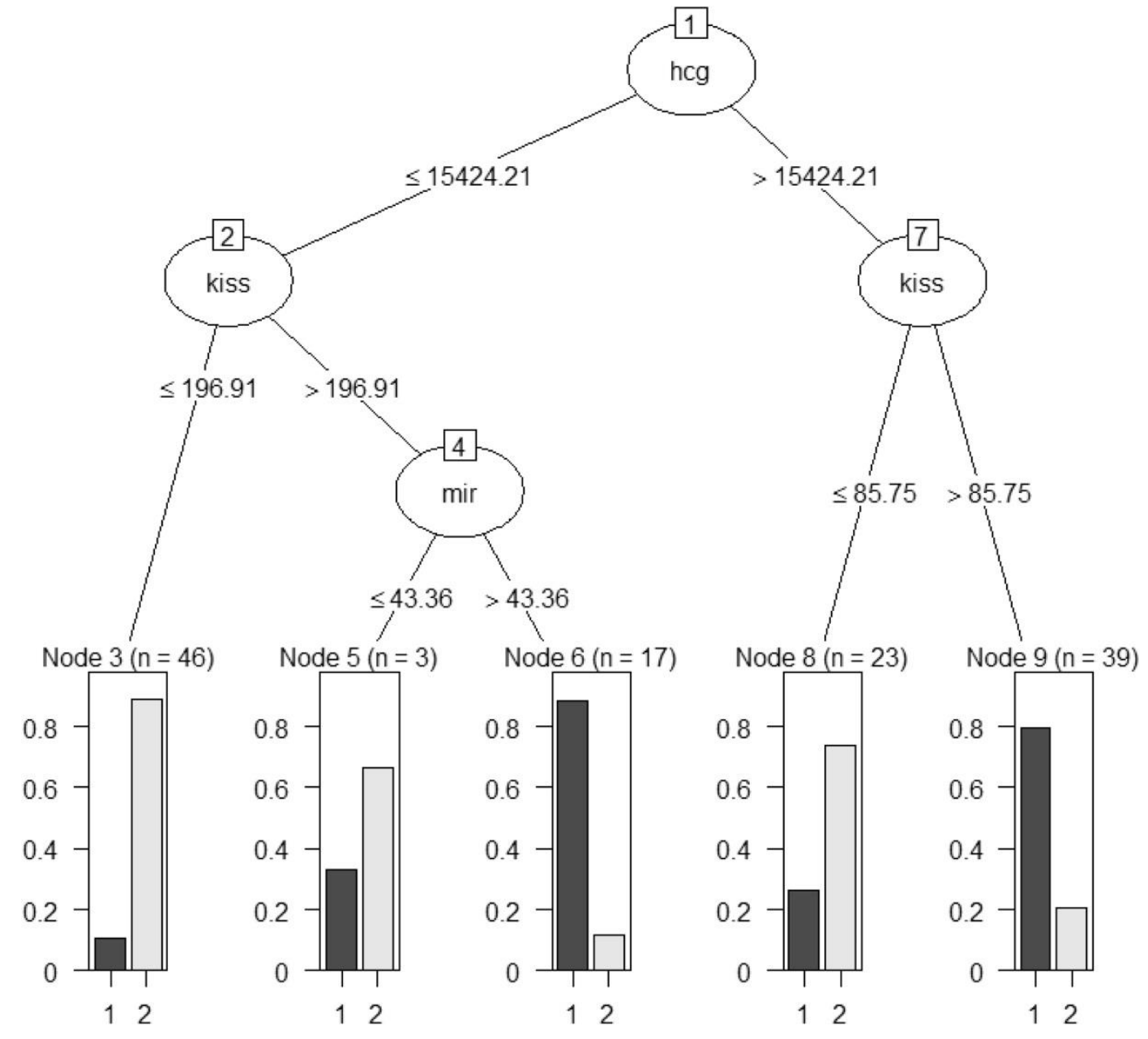

Supplemental Figure S4. Decision-tree model for triage of ectopic pregnancy

A decision-tree model that was generated with the use of a sequential combination of total human chorionic gonadotropin, kisspeptin, and miR324-3p kisspeptin levels for the prediction of ectopic pregnancy is depicted. For further details, see Methods.

$h C G$, human chorionic gonadotropin.

Romero-Ruiz et al. Kisspeptin and miR-324-3p in ectopic pregnancy. Am J Obstet Gynecol 2019. 